\title{
Understanding the phenomenology of interacting dark energy scenarios and their theoretical bounds
}

\author{
Supriya Pan, ${ }^{1, *}$ Jaume de Haro, ${ }^{2, \dagger}$ Weiqiang Yang ${ }^{3, \sharp}$ and Jaume Amorós ${ }^{2, \S}$ \\ ${ }^{1}$ Department of Mathematics, Presidency University, 86/1 College Street, Kolkata 700073, India \\ ${ }^{2}$ Departament de Matemática Aplicada, Universitat Politècnica de Catalunya, \\ Diagonal 647, 08028 Barcelona, Spain \\ ${ }^{3}$ Department of Physics, Liaoning Normal University, Dalian, 116029, People's Republic of China
}

(Received 12 February 2020; accepted 27 May 2020; published 8 June 2020)

\begin{abstract}
Nongravitational interaction between dark matter and dark energy has been considered in a spatially flat Friedmann-Lemaître-Robertson-Walker (FLRW) universe. The interaction rate is assumed to be linear in the energy densities of dark matter and dark energy and it is also proportional to the Hubble rate of the FLRW universe. This kind of interaction model leads to an autonomous linear dynamical system, and depending on the coupling parameters, could be solved analytically by calculating the exponential of the matrix, defining the system. We show here that such interaction rate has a very deep connection with some well-known cosmological theories. We then investigate the theoretical bounds on the coupling parameters of the interaction rate in order that the energy densities of the dark sector remain positive throughout the evolution of the universe and asymptotically converge to zero at very late times. Our analyses also point out that such linear interacting model may encounter with finite time future singularities depending on the coupling parameters as well as the dark energy state parameter.
\end{abstract}

DOI: $10.1103 /$ PhysRevD.101.123506

\section{INTRODUCTION}

According to recent observations [1], our Universe is currently expanding with an acceleration and this accelerating phase cannot be described by normal matter within the context of the general theory of relativity (GR). A possible approach to explain this accelerating phase of the universe within this context, i.e., within GR, is to consider some exotic matter (characterized by negative pressure), dubbed as dark energy (DE). This dark energy occupies around $68 \%$ of the total energy budget of our Universe, and this is the largest sector of our Universe [1]. However, the origin, nature and dynamics of DE are absolutely unknown even after a series of observational missions running since the last twenty years. The second largest component of our Universe is dark matter (DM) which takes nearly $28 \%$ of the total energy density of the universe, and similar to the DE sector, this sector is also not very well understood. Thus, the dynamics of our Universe is mainly driven by DE and DM, the understanding of which has been the central issue for modern cosmology at present.

In order to understand the dynamics of our Universe, mainly the dynamics of the dark sector, usually two different approaches are considered. The first approach

\footnotetext{
*supriya.maths@presiuniv.ac.in

jaime.haro@upc.edu

*d11102004@163.com

§aume.amoros@upc.edu
}

is very simple in which $\mathrm{DM}$ and $\mathrm{DE}$ are independently conserved, that means both DE and DM enjoy their independent evolution. The second approach is a bit complicated but offers a wider picture (compared to the former proposal) where DM and DE might be interacting nongravitationally with each other. Observations from many sources have already reported that a simplest cosmological model, namely the $\Lambda$-cold dark matter $(\Lambda \mathrm{CDM})$, where $\Lambda$ and $\mathrm{CDM}$ are independently conserved, is an excellent cosmological model explaining the late accelerating phase. However, in the present work, we are interested in the second approach for several reasons that we are going to describe below. If we consider the $\Lambda \mathrm{CDM}$ picture of the universe, then we are unable to explain a biggest mystery of the universe, namely, the cosmological constant problem [2]. The theory of interaction may play a very crucial role to offer a satisfactory problem to the cosmological constant problem. In [3] the author showed that a coupled system between gravity and a scalar field with exponential potential could give rise to a cosmological constant term that becomes time-dependent. When the DE era began, a new problem in the name of cosmic coincidence problem appeared. The interaction in the dark sector again played a crucial role to explain this phenomenon [4-9]. The explanations for both cosmological constant and coincidence problems influenced the scientific community to investigate interacting cosmological models, and consequently, the models in this class soon got massive 
attention due to having their far reaching activities [10-58] (see also two review articles on interacting DE models $[59,60])$. In particular, an interaction or coupling in the dark sector may naturally push a quintessence $\mathrm{DE}$, characterized by its equation of state, $w_{\mathrm{DE}}=p_{\mathrm{DE}} / \rho_{\mathrm{DE}}>-1$ to enter the phantom DE phase, $w_{\mathrm{DE}}=p_{\mathrm{DE}} / \rho_{\mathrm{DE}}<-1$ [61-63]. Here, $p_{\mathrm{DE}}$ and $\rho_{\mathrm{DE}}$ are respectively the pressure and energy density of DE. The remarkable point with such phantom crossing is that the crossing of $w_{\mathrm{DE}}=-1$ actually needs some scalar field models with negative kinetic term which automatically invites instabilities both at classical and quantum levels. But, in interacting models with the choice of suitable interaction between DE and DM, the instability problem can be relieved. Later, in the beginning of the year 2016, other observational evidences reported that the estimation of the Hubble constant, $H_{0}$, as well as, the amplitude of the matter power spectrum $\sigma_{8}$ (within the minimal $\Lambda \mathrm{CDM}$ cosmology) return different values in different observational missions that are many sigmas apart from one another. Surprisingly, DE-DM interaction again proved its potential nature by offering a possible solution to the $H_{0}$ tension $[34,37,64-67]$ and the $\sigma_{8}$ tension $[66,68-$ 70]. Certainly, based on the aforementioned limitations of the noninteracting cosmologies and the solutions coming from the interacting models, it is clear that interacting cosmologies should be investigated more elaborately in light of the above issues.

However, the first and probably the most important question in the context of interaction cosmologies is related to the energy exchange rate between the dark sectors, that means the interaction function. Since there is no globally accepted theory yet that could justify the choice of the interaction function, hence, to start with it is assumed that the interaction function maybe involve the energy densities of the dark components ranging from the simple linear to the complicated nonlinear ones. Due to such diverse choices of the interaction function one could explore a cluster of interesting possibilities as a result. However, as we will show in this work, while dealing with any interaction function, we have to be very careful because depending on the coupling parameter of interaction rate which quantifies the interaction rate in every aspect (whether the interaction rate is mild or not and the direction of energy flow it allows), the associated cosmological parameters could be unrealistic. Thus, in the present work, we show how any interaction rate can be treated leading to physically viable cosmological scenarios. We start with a simple interaction model which is linearly related to the energy densities of DM and DE as well as the Hubble rate of the Friedmann-Lemaître-Robertson-Walker universe and then discuss the theoretical bounds on this scenario and the physical consequences. The same analysis can be done for any interaction model irrespective of its linear or nonlinear functional form and consequently the bounds on such scenarios can also be imposed. The work has been organized in the following way: In Sec. II, we discuss the field equations of any interaction model and then introduce our model and provide its justifications. In Sec. III, we perform a dynamical system analysis of the original interaction model and its subcases and give the bounds on the coupling parameters for realistic interaction scenarios. In Sec. IV, we deal with future singularities, showing that for some values of the coupling parameters and the dark energy equation of state, our universe evolves to a big rip singularity. Finally, in Sec V, we conclude the main findings of the present article.

\section{INTERACTING DARK ENERGY: MODEL AND JUSTIFICATION}

In the large scale, our Universe is almost homogeneous and isotropic. The geometric configuration of this homogeneous and isotropic universe is characterized by the Friedmann-Lemaître-Robertson-Walker (FLRW) line element

$$
d s^{2}=-d t^{2}+a^{2}(t)\left[\frac{d r^{2}}{1-K r^{2}}+r^{2}\left(d \theta^{2}+\sin ^{2} \theta d \phi^{2}\right)\right],
$$

where $(t, r, \theta, \phi)$ are the comoving coordinates; $a(t)$ is the expansion scale factor of the universe; $K$ is the curvature scalar of the universe which represents a closed, open and a flat universe, respectively for its three distinct values, namely, $K=1,-1$ and 0 . Concerning the gravitational sector of our Universe, we assume that it is described by GR and the matter distribution is minimally coupled to gravity. Precisely, we consider that the total energy density of our Universe is given by, $\rho_{\text {tot }}=\rho_{r}+\rho_{b}+\rho_{c}+\rho_{x}$, where $\rho_{i}$ is the energy density of the $i$ th fluid in which $i=r, b, c, x$, respectively stands for radiation, baryons, pressure-less DM (also known as cold DM, abbreviated as CDM) and DE. The total pressure contributed due to the above components is therefore given by, $p_{\text {tot }}=p_{r}+p_{b}+$ $p_{c}+p_{x}$, where the notations follow same argument as described above. Lastly, we consider that the dark fluids of the universe namely CDM and DE are interacting nongravitationally with each other, which means that there is a continuous flow of energy and momentum between these sectors. Since other components do not interact with each other, hence, they obey their own conservation equations.

Therefore, focusing on the interacting dark sector, one can write down the conservation equations of CDM and DE as

$$
\left\{\begin{array}{l}
\dot{\rho}_{c}+3 H \rho_{c} \\
\dot{\rho}_{x}+3 H\left(1+w_{x}\right) \rho_{x}=-Q
\end{array}\right.
$$

where $-1 \leq w_{x}=p_{x} / \rho_{x}<-1 / 3$ (nonphantom fluid), is the constant equation-of-state $(\mathrm{EoS})$ parameter of the $\mathrm{DE}$ fluid and the quantity $Q$ appearing in the above two 
equations, is the energy transfer function that determines the rate of energy flow between the fluids as well as the direction of energy flow depending on its sign.

The conservation equations for the noninteracting radiation and baryonic matter are respectively

$$
\dot{\rho}_{r}+4 H \rho_{r}=0, \quad \text { and } \quad \dot{\rho}_{b}+3 H \rho_{b}=0,
$$

and introducing the time variable $N \equiv-\ln (1+z)=$ $\ln \left(a / a_{0}\right)$ which is only the number of e-folds starting at the present time, taking into account that $\dot{N}=H$, one gets

$$
\rho_{r}^{\prime}+4 \rho_{r}=0, \quad \text { and } \quad \rho_{b}^{\prime}+3 \rho_{b}=0
$$

where the prime is the derivative with respect the time $N$. The solutions have the simple form

$$
\rho_{r}=\rho_{r, e q} e^{-4\left(N-N_{e q}\right)}, \quad \text { and } \quad \rho_{b}=\rho_{b, 0} e^{-3 N},
$$

where $\rho_{r, e q}$ is the value of the radiation energy density at the matter-radiation equality and $\rho_{b, 0}$ is the present value of baryonic energy density.

Once again, in terms of the time $N$ the system of equations in (1) becomes

$$
\left\{\begin{array}{l}
\rho_{c}^{\prime}+3 \rho_{c}=-\frac{Q}{H} \\
\rho_{x}^{\prime}+3\left(1+w_{x}\right) \rho_{x}=\frac{Q}{H} .
\end{array}\right.
$$

Note that, the Hubble parameter $H$ can be found from the following equation

$$
\begin{aligned}
H^{2}(N)= & \frac{1}{3 M_{p l}^{2}}\left(\rho_{c}+\rho_{x}+\rho_{r, e q} e^{-4\left(N-N_{e q}\right)}+\rho_{b, 0} e^{-3 N}\right) \\
& -\frac{K}{a_{0}^{2} e^{2 N}},
\end{aligned}
$$

where $a_{0}$ is the present value of the scale factor. Hence, once the evolution of $\rho_{c}$ and $\rho_{x}$ are determined either analytically or numerically for some given interaction rate $Q$, the expansion rate of the universe can be determined and the modified cosmological parameters can be studied in terms of their evolution with time. Thus, as one can realize, the expansion rate of the universe is highly influenced by the interaction function. That means the expansion rate is dependent on the choice of $Q$.

In almost all works, the choice of the interaction function is motivated from the phenomenological ground. If we look at the conservation equations in (5), one can realize that the interaction rate might be the function of the energy densities of the dark sectors, namely, $\rho_{c}$ and $\rho_{x}$. Therefore, using that ground as a basis, an infinite number of interaction rates can be produced by hand and can be investigated. However, some recent investigations show that one could justify the choice of the interaction rates from some field theoretical point of view [71-76]. In particular, in [76], the authors have explicitly shown that some very well-known linear and nonlinear interaction models can be deduced from scalar field theory. Interestingly, in this work we shall show that the interaction rates can also be motivated from other well known cosmological backgrounds. We begin our discussions with a simplest interaction model of the form

$$
Q=3 H\left(\mu \rho_{c}+\nu \rho_{x}\right),
$$

where $\mu$ and $\nu$ are dimensionless coupling parameters. As one can see from (7), this interaction rate recovers some well known interaction rates as special cases. For instance, one can recover $Q=3 H \mu \rho_{c}$ under the assumption of $\nu=0$. Similarly, the model $Q=3 H \nu \rho_{x}$ is obtained when $\mu=0$. Lastly, for $\mu=\nu=\lambda$, one recovers $Q=3 H \lambda\left(\rho_{c}+\rho_{x}\right)$. One could further notice that for $\nu=-\mu$, the interaction function becomes $Q=3 H \mu\left(\rho_{c}-\rho_{x}\right)$, which has a sign changeable feature. Let us now try to justify the interaction rate (7) using the available theories in the next paragraphs.

One of the possible justifications of the interaction rate (7) may appear using the teleparallel gravity (TG), based in the Weitzenböck spacetime [77], which is equivalent to general relativity (GR) [78,79] (see also [80] which translates the early papers of Einstein about teleparalellism). Effectively, in TG to obtain the field equations the scalar torsion quantity $\mathcal{T}$ is used, which for the flat FLRW spacetime is given by $\mathcal{T}=-6 H^{2}$ [81]. Additionally, in TG the total stress tensor satisfies the conservation equation $\nabla_{\alpha} T_{\beta}^{\alpha}=0$ (see for instance [78]), where $\nabla$ denotes the usual Levi-Civita derivative. Therefore, in this framework and following [14] we consider the conservation equations in presence of an interaction as $\nabla_{\alpha} T_{\beta, A}^{\alpha}=Q_{\beta, A}$ with $A=c$, $x$, and

$$
Q_{\beta, c}=-Q_{\beta, x}=\sqrt{\mathcal{T} / 6}\left(\bar{\mu} T_{\alpha, c}^{\alpha} u_{\beta, c}+\bar{\nu} T_{\alpha, x}^{\alpha} u_{\beta, x}\right)
$$

where for a perfect fluid $T_{\beta, A}^{\alpha}=P_{A} \delta_{\beta}^{\alpha}+\left(\rho_{A}+P_{A}\right) u_{A}^{\alpha} u_{\beta, A}$ in which $u_{A}^{\alpha}=\frac{d x^{\alpha}}{\sqrt{-d s^{2}}}$ being the four-velocity of the fluid.

Then, since at the background level

$$
Q=Q_{0, c}=-Q_{0, x}=\frac{H}{\sqrt{6}}\left(\bar{\mu} \rho_{c}+\bar{\nu}\left(3 w_{x}-1\right) \rho_{x}\right),
$$

and $\nabla_{\alpha} T_{\beta, A}^{\alpha}=-\dot{\rho}_{A}-3 H\left(\rho_{A}+P_{A}\right)$, to recover our energy density transfer and the system (5), one only has to choose $\mu=\frac{\bar{\mu}}{3 \sqrt{6}}$ and $\nu=\frac{\left(3 w_{x}-1\right) \bar{\nu}}{3 \sqrt{6}}$.

On the other hand, following the notation of [14] the perturbed four-velocity of the B-fluid is given by $u_{B}^{\alpha}=\left(1-\phi, \frac{1}{a} \partial^{i} v_{B}\right)$, where $\phi$ is the Newtonian potential, $v_{B}$ is the particular velocity and $B=r, b, c, x$. This means that at the background level the four-velocity, in the comoving system, becomes $u_{B}^{\alpha}=(1,0,0,0)$ for any 
fluid which is the consequence of the isotropy at the background level. Therefore, at the background level the scalar $u_{\alpha, B} T_{\beta, B}^{\alpha} u^{\beta}$ becomes $\rho_{B}$, and one can also choose the energy transfer function

$Q_{\beta, c}=-Q_{\beta, x}=3 \sqrt{\mathcal{T} / 6}\left(\mu u_{\alpha, c} T_{\gamma, c}^{\alpha} u_{c}^{\gamma} u_{\beta, c}+\nu u_{\alpha, x} T_{\gamma, x}^{\alpha} u_{x}^{\gamma} u_{\beta, x}\right)$.

Following this way, and now working in the framework of GR, we can consider, for example, the scalar $\nabla_{\alpha} u_{c}^{\alpha}$ which for the flat FLRW metric leads to $3 H$, and thus one may consider

$Q_{\beta, c}=-Q_{\beta, x}=\nabla_{\eta} u_{c}^{\eta}\left(\mu u_{\alpha, c} T_{\gamma, c}^{\alpha} u_{c}^{\gamma} u_{\beta, c}+\nu u_{\alpha, x} T_{\gamma, x}^{\alpha} u_{x}^{\gamma} u_{\beta, x}\right)$,

or

$$
Q_{\beta, c}=-Q_{\beta, x}=\frac{1}{3} \nabla_{\eta} u_{c}^{\eta}\left(\bar{\mu} T_{\alpha, c}^{\alpha} u_{\beta, c}+\bar{\nu} T_{\alpha, x}^{\alpha} u_{\beta, x}\right) .
$$

In addition, in GR one could use the CarminatiMcLenaghan invariants [82]

$$
\mathcal{R}_{2} \equiv \frac{1}{4} \mathcal{R}_{\mu}^{\nu} \mathcal{R}_{\nu}^{\mu}, \quad \text { and } \quad \mathcal{R}_{3} \equiv-\frac{1}{8} \mathcal{R}_{\mu}^{\nu} \mathcal{R}_{\gamma}^{\mu} \mathcal{R}_{\nu}^{\gamma},
$$

whose values for the synchronous comoving observers in the flat FLRW space-time are given by $\mathcal{R}_{2}=\frac{3}{4} \dot{H}^{2}$ and $\mathcal{R}_{3}=-\frac{3}{8} \dot{H}^{3}$. Thus, in such coordinates, one finds that, $\dot{H}=-2 \frac{\mathcal{R}_{3}}{\mathcal{R}_{2}}$, and consequently, the scalar curvature, namely $R$, takes the relation $H^{2}=\frac{\mathcal{R}_{3}}{\mathcal{R}_{2}}+\frac{R}{12}$. Following this, one can replace $\nabla_{\eta} u_{c}^{\eta}$ by the following scalar a

$$
\mathcal{R} \equiv 3 \sqrt{\frac{\mathcal{R}_{3}}{\mathcal{R}_{2}}+\frac{R}{12}},
$$

in the Eqs. (11) and (12). Finally, going beyond GR one can use a mimetic gravity, based on a mimetic field, namely $\varphi$, satisfying $\nabla_{\alpha} \varphi \nabla^{\alpha} \varphi=-1$ (see [83,84] for a detailed description of this theory). Thus, in mimetic gravity the scalar $\chi=-\nabla^{\alpha} \nabla_{\alpha} \varphi$ in the flat FLRW space-time becomes $3 H$. Thus, one can see that the present interaction model can be justified using various well-known cosmological theories.

Let us note that although the interaction rate (7) is already existing in the list of some well-known linear interaction rates, however, below we shall show why we should reconsider the same model.

To conclude this section we note that the system of first order differential equations given in (5) depicts a two dimensional autonomous dynamical system, which could be solved once one has the values of the corresponding energy densities at present time.

\section{DYNAMICAL SYSTEM ANALYSIS}

In this section we shall perform a detailed dynamical analysis of the interaction model (7) as well as the simple cases of (7) aiming to provide with the bounds on the coupling parameters of this interaction model. Let us start our analysis with the general interaction model (7) for which the dynamical system becomes

$$
X^{\prime}=A X
$$

where prime, as already mentioned earlier, denotes the differentiation with respect to $N$ and

$A=\left(\begin{array}{cc}-3(\mu+1) & -3 \nu \\ 3 \mu & -3\left(1+w_{x}-\nu\right)\end{array}\right), \quad X=\left(\begin{array}{c}\rho_{c} \\ \rho_{x}\end{array}\right)$.

Since we are dealing with a non-degenerate linear dynamical system, the only fixed point is the origin, and its stability is deduced using the trace-determinant criterion. Physically, we want that the origin is an attractor, because in the contrary case we will have future singular solutions, so we have to impose the condition $\operatorname{Tr} A=$ $-3\left(2+\mu-\nu+w_{x}\right)<0$ and $\operatorname{Det} A=9(\mu+1)\left(1+w_{x}-\nu\right)+$ $9 \mu \nu>0$. These lead to the allowed region in the plane of parameters $(\mu, \nu)$ determined by the linear inequalities

$$
\left\{\begin{array}{l}
\nu<2+\mu+w_{x}, \\
\nu<\left(1+w_{x}\right)(\mu+1),
\end{array}\right.
$$

which involves the EoS of DE along with the coupling parameters. In particular, if the DE is assumed to be the vacuum energy characterized by $w_{x}=-1$, the above domain becomes

$$
D=\{(\mu, \nu): \mu>\nu-1, \nu<0\} .
$$

However, the condition that the origin is an attractor is not enough, because if the origin is an attractor focus, the orbits will round around $(0,0)$, and thus, the energy densities will be negative, which has no physical sense. For this reason we have to demand that the discriminant $\Delta=(\operatorname{Tr} A)^{2}-4 \operatorname{Det} A$ has to be positive, that means,

$$
\Delta=9\left(\left(w_{x}-\mu-\nu\right)^{2}-4 \mu \nu\right)>0 .
$$

In addition, if we want that the energy densities must be positive all the time, then we also need to demand that the eigenvectors of $A$, namely $\vec{v}_{ \pm}=\left(v_{ \pm, 1}, v_{ \pm, 2}\right)$, have to stay in the first quadrant, i.e., they have to satisfy $v_{ \pm, 1} v_{ \pm, 2} \geq 0$. Effectively, since the system is autonomous, the orbits never cross, then with this condition, the orbits 
$\left\{e^{\lambda_{+} N} \vec{v}_{+}\right\}_{N \in \mathbb{R}}$ and $\left\{e^{\lambda_{-} N} \vec{v}_{-}\right\}_{N \in \mathbb{R}}$ define a sector in the first quadrant, and all solution with initial conditions in this sector defines an orbit inside it.

Denoting the eigenvalues of the matrix $A$ by $\lambda_{ \pm}=$ $(\operatorname{Tr} A \pm \sqrt{\Delta}) / 2$, the corresponding eigenvectors are given by as follows:

(1) For $\nu \neq 0$ :

$$
\vec{v}_{ \pm}=\left(1,-\frac{\mu+1+\lambda_{ \pm} / 3}{\nu}\right)
$$

and thus, the condition $v_{ \pm, 1} v_{ \pm, 2} \geq 0$, becomes

$$
\frac{\mu+1+\lambda_{ \pm} / 3}{\nu} \leq 0
$$

Then, to have positive energy densities all the time, the initial condition $\left(\rho_{c, 0}, \rho_{x, 0}\right)$ has to satisfy

$$
\begin{aligned}
& \min \left(-\frac{\mu+1+\lambda_{+} / 3}{\nu},-\frac{\mu+1+\lambda_{-} / 3}{\nu}\right) \\
& \leq \frac{\rho_{0, x}}{\rho_{0, c}} \leq \max \left(-\frac{\mu+1+\lambda_{+} / 3}{\nu},-\frac{\mu+1+\lambda_{-} / 3}{\nu}\right) .
\end{aligned}
$$

(2) For $\nu=0$ : The eigenvalues are $\lambda_{+}=-3\left(1+w_{x}\right)$ and $\lambda_{-}=-3(\mu+1)$, and the corresponding eigenvectors are given by

$$
\vec{v}_{+}=(0,1), \quad \vec{v}_{-}=\left(1, \frac{\mu}{w_{x}-\mu}\right)
$$

and thus, the condition $v_{ \pm, 1} v_{ \pm, 2} \geq 0$, becomes $w_{x} \leq \mu \leq 0$.

Then, the condition to have positive energy densities all the time is

$$
\frac{\rho_{x, 0}}{\rho_{c, 0}} \geq \frac{\mu}{w_{x}-\mu} .
$$

Taking into account that $\frac{\rho_{x, 0}}{\rho_{c, 0}}=\frac{\Omega_{x, 0}}{\Omega_{c, 0}}$, and as we will see, if one disregards the energy of the radiation at the present time one has $\Omega_{c, 0} \cong 0.262$ and $\Omega_{x, 0} \cong 0.69$, hence, the condition (24) becomes $\mu \geq 0.72 w_{x}$, which means that the parameter $\mu$ is constrained to satisfy

$$
0.72 w_{x} \leq \mu \leq 0
$$

On the other hand, to know the value of the effective EoS parameter and thus, to know if the Universe accelerates or decelerates, we need to calculate explicitly the solutions of (15) which is given by $X(N)=e^{A N} X_{0}, X_{0}$ being the current value of $X$ with

$$
e^{A N}=B\left(\begin{array}{cc}
e^{\lambda_{+} N} & 0 \\
0 & e^{\lambda_{-} N}
\end{array}\right) B^{-1}
$$

where, $\lambda_{ \pm}$are once again the eigenvalues and the matrix $B$ is set up with the eigenvectors of $A, \vec{v}_{ \pm}$, i.e.,

$$
B=\left(\begin{array}{ll}
v_{+, 1} & v_{-, 1} \\
v_{+, 2} & v_{-, 2}
\end{array}\right) .
$$

Thus, when $\nu \neq 0$, since $\operatorname{Det} B=\frac{\sqrt{\Delta}}{3 \nu}$, we will have

$$
B=\left(\begin{array}{cc}
1 & 1 \\
v_{+, 2} & v_{-, 2}
\end{array}\right), \quad B^{-1}=\frac{3 \nu}{\sqrt{\Delta}}\left(\begin{array}{cc}
v_{-, 2} & -1 \\
-v_{+, 2} & 1
\end{array}\right)
$$

and consequently,

$e^{A N}=\frac{3 \nu}{\sqrt{\Delta}}\left(\begin{array}{cc}e^{\lambda_{+} N} v_{-, 2}-e^{\lambda_{-} N} v_{+, 2} & e^{\lambda_{-} N}-e^{\lambda_{+} N} \\ v_{+, 2} v_{-, 2}\left(e^{\lambda_{+} N}-e^{\lambda_{-} N}\right) & e^{\lambda_{-} N} v_{-, 2}-e^{\lambda_{+} N} v_{+, 2}\end{array}\right)$.

This could be written in terms of the discriminant as follows

$$
\begin{aligned}
e^{A N}= & \frac{e^{-\frac{3}{2}\left(2+w_{x}-\nu+\mu\right) N}}{\sqrt{\Delta}}\left[\left(\begin{array}{cc}
\sqrt{\Delta} \cosh \left(\frac{\sqrt{\Delta} N}{2}\right) & -6 \nu \sinh \left(\frac{\sqrt{\Delta} N}{2}\right) \\
6 \mu \sinh \left(\frac{\sqrt{\Delta} N}{2}\right) & -\sqrt{\Delta} \cosh \left(\frac{\sqrt{\Delta} N}{2}\right)
\end{array}\right)\right. \\
& \left.+3\left(w_{x}-\nu-\mu\right) \sinh \left(\frac{\sqrt{\Delta} N}{2}\right) \mathrm{Id}\right],
\end{aligned}
$$

where Id denotes the identity matrix.

Finally, about the initial conditions it is useful to introduce the variables $\bar{\rho}_{i}=\frac{\rho_{i}}{3 H_{0}^{2} M_{p l}^{2}}$ with $i=r, b, c, x$. Then, in the flat case $K=0$, the initial conditions are $\Omega_{i, 0}$. We could choose the central value of $\Omega_{m, 0}=0.31$ for the total matter (baryonic and dark) sector of the universe. Using the observational estimation of $\Omega_{b, 0} h^{2}=0.0221$ and $\Omega_{c, 0} h^{2}=0.1206$ we see that the percent of baryonic matter is approximately the $15.5 \%$ of the total matter, so $\bar{\rho}_{b, 0}=\Omega_{b, 0}=0.048$ and $\bar{\rho}_{c, 0}=\Omega_{c, 0}=0.262$. Since, at the present time, the energy density of radiation is negligible compared to other energy densities, one can approximately take $\bar{\rho}_{x, 0}=\Omega_{x, 0}=1-\Omega_{b, 0}-\Omega_{c, 0} \cong 0.69$.

To obtain the evolution of the energy density of radiation we take, for example, the redshift at the matter radiation-equality equal to its central value $z_{e q}=3411$ for the PlanckTT + lowE likelihood [85], for which $N_{e q}=$ -8.135 , and thus, from the matter-radiation equality,

$\bar{\rho}_{r, e q} \equiv \bar{\rho}_{r}\left(N_{e q}\right)=\bar{\rho}_{b}\left(N_{e q}\right)+\bar{\rho}_{c}\left(N_{e q}\right)=\bar{\rho}_{b, 0} e^{-3 N_{e q}}+\bar{\rho}_{c}\left(N_{e q}\right)$, 
once the parameters $\mu, \nu$ and $w_{x}$, are fixed, one obtains $\bar{\rho}_{r}\left(N_{e q}\right)=\bar{\rho}_{r, e q} e^{-4\left(N-N_{e q}\right)}$. And when the initial conditions are obtained one can easily calculate $\bar{\rho}_{i}(N)$ and also the effective equation of state $(\mathrm{EoS})$ parameter defined by $w_{\text {eff }}(N)=\frac{\rho_{\text {tot }}}{p_{\text {tot }}}=\frac{w_{x} \bar{\rho}_{x}(N)+\frac{1}{3} \bar{\rho}_{r}(N)}{\bar{\rho}_{\text {tot }}(N)}$.

\section{A. Special cases}

In this section we consider the special cases emerging from the original interaction (7). For example, one of the simplest cases that one may consider the case with $\nu=0$ in (7) which returns, $Q=3 H \mu \rho_{c}$. Similarly, one could equally consider another case with $\mu=0$, equivalently, the interaction rate becomes $Q=3 H \nu \rho_{x}$. The equality $\mu=\nu$ is also interesting. The solutions for all the cases are trivial, however, there is something that needs to be clarified in this article for future works. In what follows, we describe the solutions for each model as well as the bounds on the

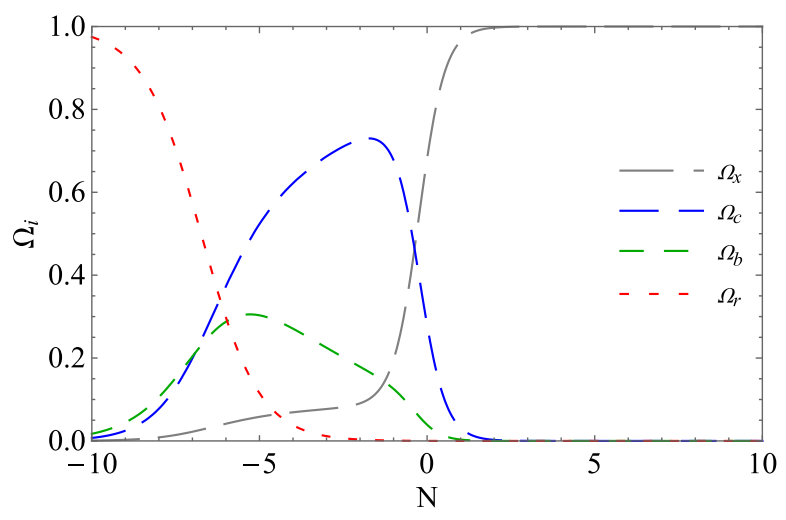

FIG. 1. The evolution of the density parameters namely, $\Omega_{r}$ (red curve), $\Omega_{b}$ (green curve), $\Omega_{c}$ (blue curve) and $\Omega_{x}$ (grey curve) for the interaction rate (7) with $\nu=0, \mu=0.1 w_{x}, w_{x}=-0.95$ has been shown in this picture.

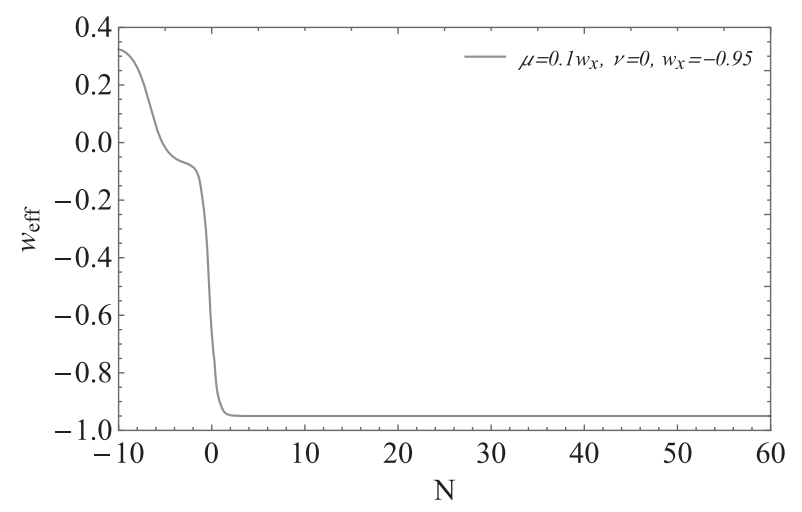

coupling parameters for which one obtains viable cosmological solutions.

(1) For the interaction rate, $Q=3 H \mu \rho_{c}$, the condition (25) requires $0.72 w_{x} \leq \mu \leq 0$, and the solution, which is non-singular and positive, is given by

$$
\begin{aligned}
\bar{\rho}_{c}(N)= & \Omega_{c, 0} e^{-3(1+\mu) N}, \quad \text { and } \\
\bar{\rho}_{x}(N)= & \Omega_{x, 0} e^{-3\left(1+w_{x}\right) N} \\
& -\frac{\mu \Omega_{c, 0}}{\mu-w_{x}}\left(e^{-3(1+\mu) N}-e^{-3\left(1+w_{x}\right) N}\right) .
\end{aligned}
$$

Finally, with this quantities one easily has the total pressure and energy density, so we have completely determined the effective EoS parameter $w_{\text {eff }}(N)$. It is easy to see that $w_{\text {eff }}(N) \rightarrow w_{x}$ when $N \rightarrow \infty$. To understand the evolution of various components in terms of their energy densities in Fig. 1, we display them for a specific choice of the coupling parameter, $\mu$ constrained in the region $0.72 w_{x} \leq \mu \leq 0$ and for a particular choice of the EoS of DE, $w_{x}$. As one can see, all the energy densities remain positive for this choice of the coupling parameter. In Fig. 2, we also show the evolution of the effective EoS, $w_{\text {eff }}$ for the same value of $\mu$ and $w_{x}$ used to draw Fig. 1 . To be precise, in the left panel of Fig. 2, we show the evolution of $w_{\text {eff }}$ for a wide region where $N \in$ $[-10,60]$ and in the right panel of Fig. 2 we show the evolution of $w_{\text {eff }}$ from the epoch of matterradiation equality to present time. One can clearly visualize from Fig. 2, that $w_{\text {eff }}$ crosses from positive (in the early phase of the universe) to negative values (at present time) and then asymptotically converges to $w_{x}=-0.95$.

(2) For the second case with $\mu=0$, equivalently for the interaction rate $Q=3 H \nu \rho_{x}$, working in the same way as above, one obtains $\lambda_{+}=-3\left(1+w_{x}-\nu\right)$ and $\lambda_{-}=-3$, and thus,

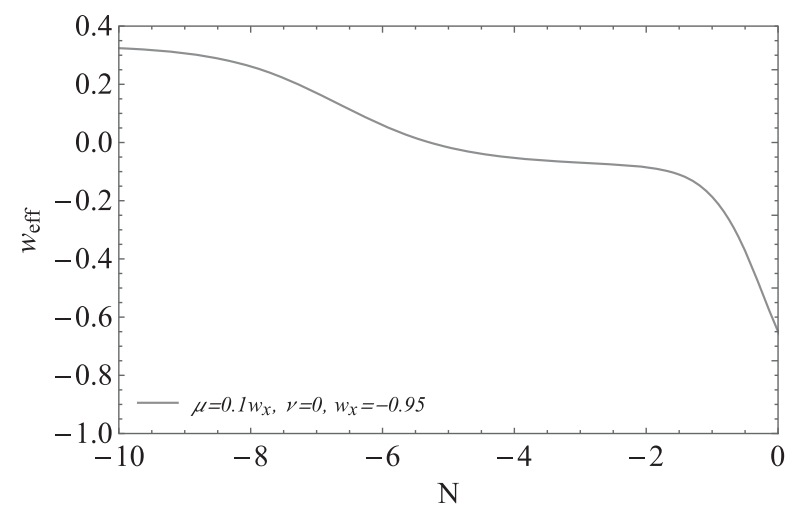

FIG. 2. We show the evolution of the effective EoS of DE for the interaction scenario with $\nu=0, \mu=0.1 w_{x}, w_{x}=-0.95$. In the left panel we see the asymptotic evolution of $w_{\text {eff }}$ for the region $N \in[-10,60]$, and in the right panel we only show its evolution from the matter-radiation equality to the present time, that means for $N \in[-10,0]$. One can notice that the effective equation of state parameter converges to $w_{x}=-0.95$. 


$$
v_{+}=\left(1, \frac{w_{x}-\nu}{\nu}\right) \quad v_{-}=(1,0)
$$

In that case the condition $v_{ \pm, 1} v_{ \pm, 2} \geq 0$ and $\frac{\rho_{x, 0}}{\rho_{c, 0}} \leq$ $\frac{w_{x}-\nu}{\nu}$ requires $0.27 w_{x} \leq \nu \leq 0$, and the solution is given by

$$
\begin{aligned}
& \bar{\rho}_{x}(N)=\Omega_{x, 0} e^{-3\left(1+w_{x}-\nu\right) N} \text { and } \\
& \bar{\rho}_{c}(N)=\Omega_{c, 0} e^{-3 N}+\frac{\nu \Omega_{x, 0}}{w_{x}-\nu}\left(e^{-3\left(1+w_{x}-\nu\right) N}-e^{-3 N}\right) .
\end{aligned}
$$

Finally, a simple calculation shows that

$$
\lim _{N \rightarrow \infty} w_{\text {eff }}(N) \equiv w_{\text {eff }, \infty}=w_{x}-\nu \geq w_{x},
$$

but this does not mean that the universe could decelerate once again. Effectively, at the present

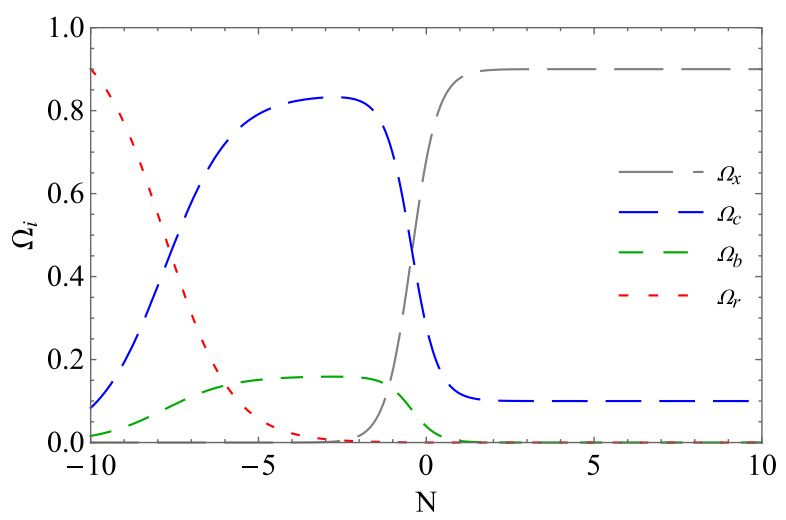

FIG. 3. The evolution of the density parameters namely, $\Omega_{r}$ (red curve), $\Omega_{b}$ (green curve), $\Omega_{c}$ (blue curve) and $\Omega_{x}$ (grey curve) for the interaction rate (7) with $\mu=0, \nu=0.1 w_{x}$, and $w_{x}=-0.95$ has been displayed.

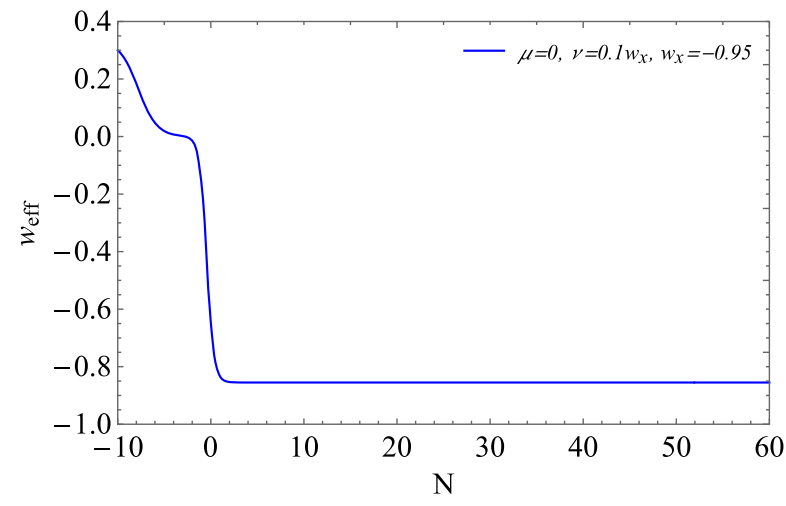

time we have $w_{\text {eff }, 0} \cong w_{x} \Omega_{x, 0}$ because the radiation is negligible. Then, since nowadays our universe is accelerating, hence, $w_{\text {eff }, 0}$ has to be less than $-1 / 3$ and taking a typical value of the density parameter for $\mathrm{DE}$ as $\Omega_{x, 0} \cong 0.69$, this means that $-1 \leq w_{x}<$ 0.483 , and thus,

$$
\begin{aligned}
& w_{\text {eff, },}<(1-0.27) w_{x} \\
& =0.73 w_{x}<-0.73 \times 0.483 \cong-0.35<-1 / 3,
\end{aligned}
$$

meaning that the universe accelerates at late times. Similarly, for this special case too, we have calculated the density parameters of different cosmic fluids as well as the effective EoS of the total fluid. In Fig. 3, we show the density parameters for a specific choice of the coupling parameter, $\nu$ satisfying the constraint $0.72 w_{x} \leq \nu \leq 0$ and for a particular choice of the EoS of DE, $w_{x}=-0.95$. Additionally, in Fig. 4, we depict the evolution of the effective EoS, $w_{\text {eff }}$ for the same value of $\nu$ and $w_{x}$ used to draw Fig. 3. In the left panel of Fig. 4, we describe the evolution of $w_{\text {eff }}$ for $N \in[-10,60]$ while in the right panel of Fig. 4 we show the evolution of $w_{\text {eff }}$ from the epoch of matter-radiation equality to present time. A clear transition of $w_{\text {eff }}$ from its positive values to negative values are found from Fig. 4.

Remark 1: We would like remark that to obtain a viable cosmological scenario driven by the linear interaction models prescribed above, one needs to impose the condition $0.27 w_{x} \leq \nu($ or $\mu) \leq 0$. However, sometimes this condition is somehow overlooked and due to arbitrary choice of the coupling parameters, the energy densities of the dark sector do not remain positive throughout the evolution of the universe. Sometimes a negative sign is considered in the interaction rate which leads to additional confusions. Thus, we would like to clarify this point in our

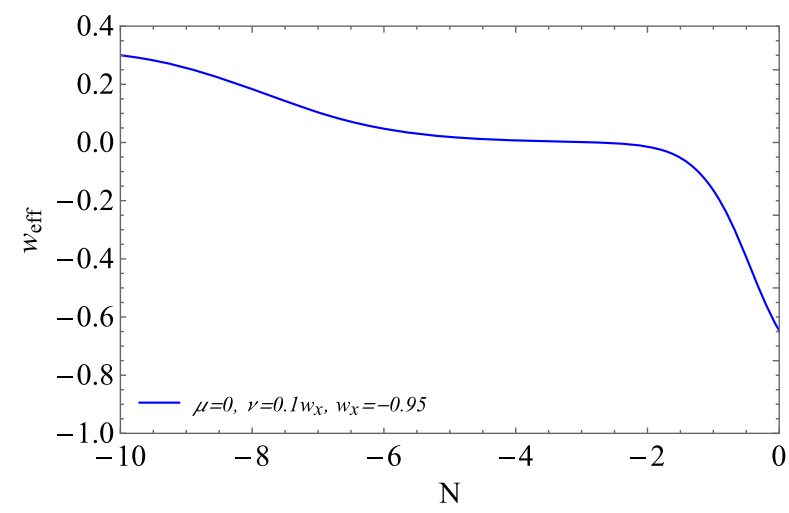

FIG. 4. We display the evolution of the effective EoS of DE for the interaction rate (7) with $\mu=0, \nu=0.1 w_{x}, w_{x}=-0.95$. In the left panel we see the asymptotic evolution of $w_{\text {eff }}$ for the region $N \in[-10,60]$, and in the right panel we only show its evolution from the matter-radiation equality to the present time, that means for $N \in[-10,0]$. One can notice that the effective equation of state parameter converges to $w_{x}-\nu=-0.855$ in an asymptotic manner. 
notation. For instance, let us select the interaction rate $Q=-\xi H \rho_{x}$, which in our notation implies, $\xi=-3 \nu$. Now, since $0.27 w_{x} \leq \nu \leq 0$, hence, we need to choose positive values of $\xi$. The energy densities become positive for times previous to the present time, but as we can see from (34) the energy density of the dark matter becomes negative at late times. Alternatively, if we select $Q=\alpha H \rho_{c}$ which in our notation $\mu=\alpha / 3$. Again we need to choose $\alpha<0$ because for positive values of $\alpha$, the energy density of the dark energy $\rho_{x}$ becomes negative at early times, meaning that no viable cosmic scenario for these parameters.

(3) Another theoretically interesting case might be the one when the coupling parameters are equal, that means $\mu=\nu$. In this situation the trace is given by $\operatorname{Tr} A=-3\left(2+w_{x}\right)$, and it is negative because we are considering non-phantom fluids. The determinant is given by $\operatorname{Det} A=9\left(1+w_{x}+\mu w_{x}\right)$, and it is positive when $\mu<-1-\frac{1}{w_{x}}$. For the discriminant one has $\Delta=9 w_{x}\left(w_{x}-4 \mu\right)$, meaning that it is positive for $\mu>w_{x} / 4$. So, for the moment we have

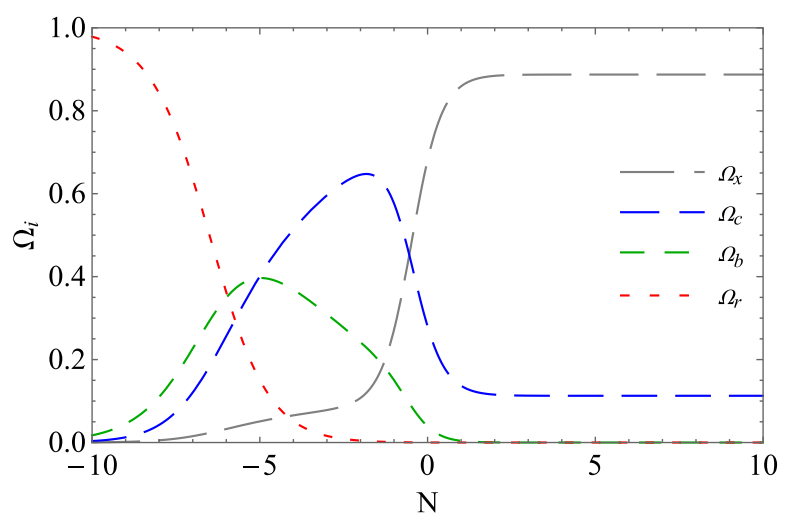

FIG. 5. The evolution of the density parameters, namely, $\Omega_{r}$ (red curve), $\Omega_{b}$ (green curve), $\Omega_{c}$ (blue curve), and $\Omega_{x}$ (grey curve) for the interaction rate (7) with $\mu=\nu=0.1 w_{x}$ and $w_{x}=$ -0.95 has been shown.

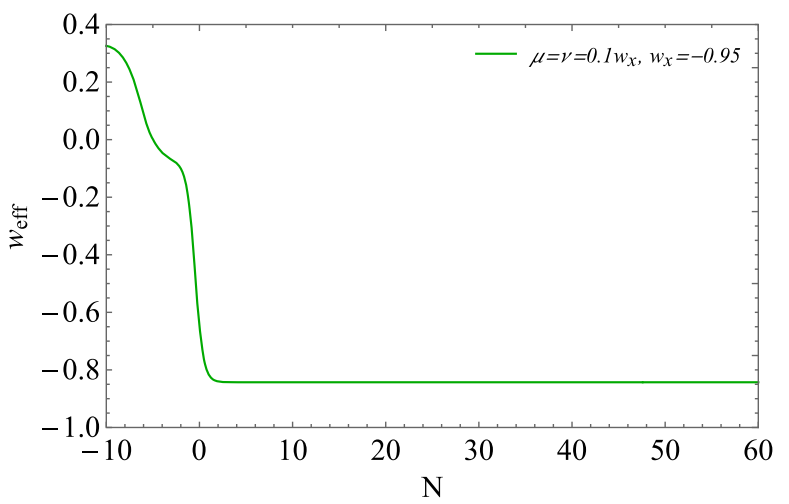

$w_{x} / 4<\mu<-\left(w_{x}+1\right) / w_{x}$. Now we deal with the condition (21). Since

$\lambda_{ \pm}=-\frac{3\left(2+w_{x}\right)}{2} \pm \frac{3}{2} \sqrt{\left(2 \mu-w_{x}\right)^{2}-4 \mu^{2}}$,

the constraint (21) becomes

$$
\frac{1}{2 \mu}\left(2 \mu-w_{x} \pm \sqrt{\left(2 \mu-w_{x}\right)^{2}-4 \mu^{2}}\right) \leq 0,
$$

which is satisfied only for $w_{x} / 2 \leq \mu \leq 0$. Then, together with $w_{x} / 4<\mu<-\left(w_{x}+1\right) / w_{x}$, we will have

$$
w_{x} / 4<\mu \leq 0 \text {. }
$$

Finally, the condition (22) becomes

$$
\begin{aligned}
& -\frac{1}{2 \mu}\left(2 \mu-w_{x}-\sqrt{\left(2 \mu-w_{x}\right)^{2}-4 \mu^{2}}\right) \\
& \leq \frac{\rho_{x, 0}}{\rho_{c, 0}} \leq-\frac{1}{2 \mu}\left(2 \mu-w_{x}+\sqrt{\left(2 \mu-w_{x}\right)^{2}-4 \mu^{2}}\right) .
\end{aligned}
$$

Now since $\frac{\rho_{x, 0}}{\rho_{c, 0}}=\frac{\Omega_{x, 0}}{\Omega_{c, 0}} \cong 2.63$ we obtain the following bound

$$
0.18 w_{x} \leq \mu \leq 0 \Rightarrow-0.18 \leq \mu \leq 0
$$

because as already mentioned, we are dealing with nonphantom fields, i.e., $-1 \leq w_{x}<-1 / 3$. For this interaction model we have similarly shown the modified density parameters in Fig. 5 and the effective equation of state parameter in Fig. 6 taking the choices $\mu=n u=0.1 w_{x}$ with $w_{x}=-0.95$. Finally, we have considered a noninteracting scenario of the universe, equivalently, $\mu=\nu=0$ and shown the density parameters as well as the effective

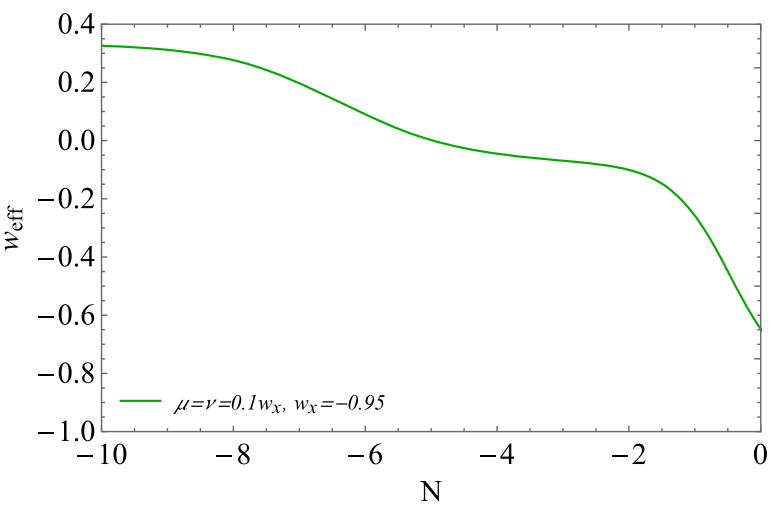

FIG. 6. We display the evolution of the effective EoS of DE for the interaction rate (7) with $\mu=\nu=0.1 w_{x}, w_{x}=-0.95$. In the left panel we show the asymptotic evolution of $w_{\text {eff }}$ for the region $N \in[-10,60]$, and in the right panel we only show its evolution from the matter-radiation equality to the present time, that means for $N \in[-10,0]$. 


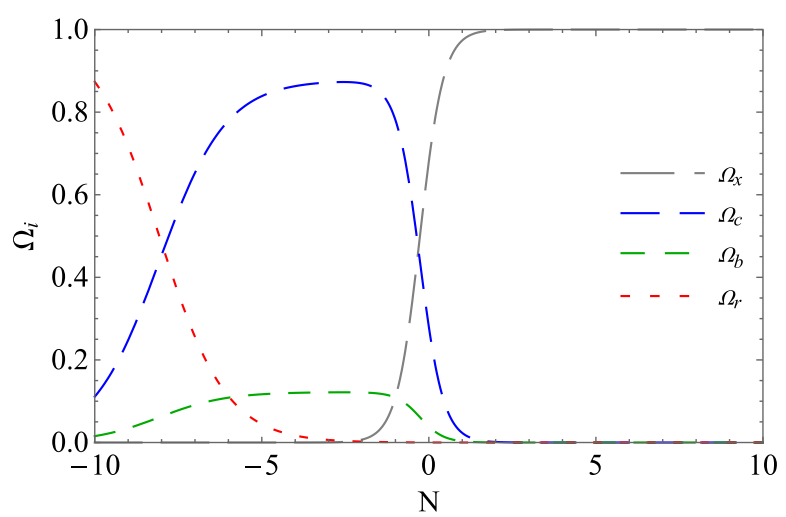

FIG. 7. We show the evolution of the density parameters, namely, $\Omega_{r}$ (red curve), $\Omega_{b}$ (green curve), $\Omega_{c}$ (blue curve), and $\Omega_{x}$ (grey curve) for the non-interacting case $\mu=\nu=0$, with $w_{x}=-0.95$.

equation of state in Figs. 7 and 8, respectively, taking a specific value of $w_{x}=-0.95$. The Figs. 7 and 8 are motivated to present a comparison between various interacting scenarios with the non-interacting one.

It is well known that in the noninteracting case the perturbations grow in a matter-dominated regime, i.e., when $w_{\text {eff }}=0$ and the physical scales reenter the Hubble horizon $k^{2} \gg a^{2} H^{2}$ [86] (see also [87] where the authors, using a matrix approach, show that, in the noninteracting case, the dominant perturbations become constant in the super-horizon scales during the radiation era, and for an interacting case, see [88], where the authors, following the same analysis as in [87], arrive at the same conclusion). So, a matter-dominated state is necessary after the radiation domination and before the present time. Although this is a topic that deserves future investigations, we hope that the same will happen in the interacting case for values of the parameters $\mu$ and $\nu$ close to zero. For this reason, comparing the different effective equation of state parameters studied here, we see that the best case is when $\mu=0$ and $\nu=0.1 w_{x}$ with $w_{x}=-0.95$.

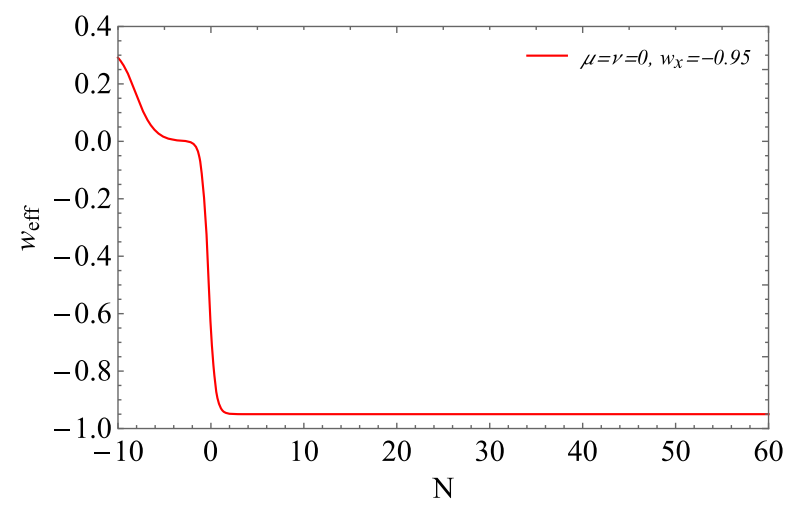

In fact, studies in [14] agree that interacting models may lead to early time instabilities where the instabilities of the perturbations are only considered in superhorizon scales and extrapolated (see for instance Eqs. (63)-(75) of [14]) to subhorizon scales. However, why one can extrapolate from superhorizon to subhorizon scales, is not clear, and hence, the instability problem in this context does not seem to be conclusive and this deserves further investigations. Moreover, when obtaining perturbations many orders greater than the background, it has not been realized that the linear approximation only holds for perturbations less or the same order of the background. More interesting are the conclusions of [89], where the authors state the difficulty of the study of subhorizon perturbations because it is impossible, in this regime, to find analytic solutions, and the only conclusion is that the perturbations grow slower than in the noninteracting case. The understanding of early time instabilities appearing in the interacting DE-DM theories is therefore a key topic that deserves serious attention. Such instabilities are highly model dependent since it can be avoided with the proper choice of the interaction function [88]. So, one may argue that the instabilities appearing in such theories point toward the insufficiencies of the phenomenological parametrizations of the interaction functions. Although the linear interaction model is the most simplest and convenient choice to proceed with, however, one may consider suitable parametrizations of the interaction functions beyond the linear parametrizations with an aim to investigate the evolution of the interacting universe at the level of perturbations.

\section{FUTURE SINGULARITIES}

In this section we demonstrate that the interaction scenarios may lead to finite time singularities. In particular, we find that our present linear interacting model may also lead to finite time future singularities. Effectively, if one takes $\operatorname{Det} A=9(\mu+1)\left(1+w_{x}-\nu\right)+9 \mu \nu<0$, the origin becomes a saddle point, and thus, at very late times, the

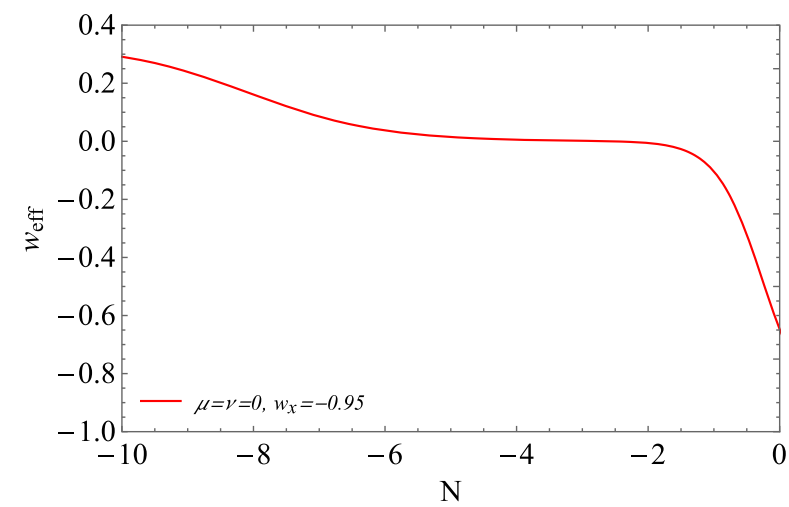

FIG. 8. We display the evolution of the effective EoS of DE for the noninteraction scenario that means with $\mu=\nu=0$, and $w_{x}=-0.95$. In the left panel we show the asymptotic evolution of $w_{\text {eff }}$ for the region $N \in[-10,60]$, and in the right panel we only show its evolution from the matter-radiation equality to the present time, that means for $N \in[-10,0]$. 
total energy density diverges, meaning that the universe will enter in a phantom dominated phase finishing in a big rip singularity. To show that, we choose for example, $\mu=0$ which implies $1+w_{x}-\nu<0$ and this restriction together with the condition $\frac{\rho_{x, 0}}{\rho_{c, 0}} \leq \frac{w_{x}-\nu}{\nu}$ leads to the constraint

$$
\max \left(0.27 w_{x}, 1+w_{x}\right)<\nu<0,
$$

which implies that $w_{x}<-1$, that means, effectively $\rho_{x}$ must be a phantom fluid. Thus, at late times, the solution (34) becomes

$$
\begin{aligned}
& \bar{\rho}_{x}(N) \sim \Omega_{x, 0} e^{-3\left(1+w_{x}-\nu\right) N} \bar{\rho}_{x}(N) \rightarrow \infty, \quad \text { and } \\
& \bar{\rho}_{c}(N) \sim \frac{\nu}{w_{x}-\nu} \bar{\rho}_{x}(N) \bar{\rho}_{x}(N) \rightarrow \infty
\end{aligned}
$$

and $w_{\text {eff, } \infty}=w_{x}-\nu<-1$, which means that, at late times, the universe enters into a phase dominated by the phantom fluid.

Finally, at very late times, the Raychaudhuri equation for $K=0$ universe becomes

$$
\dot{H}=-\frac{1}{2 M_{p l}^{2}}\left(1+w_{\mathrm{eff}, \infty}\right) \rho_{\mathrm{tot}}=-\frac{3}{2}\left(1+w_{\mathrm{eff}, \infty}\right) H^{2},
$$

whose solution is given by

$$
\begin{aligned}
& \frac{1}{H_{0}}-\frac{1}{H}=-\frac{3}{2}\left(1+w_{\mathrm{eff}, \infty}\right)\left(t-t_{0}\right) \\
& \Leftrightarrow H(t)=\frac{2}{3\left(1+w_{\mathrm{eff}, \infty}\right)} \frac{1}{t-t_{s}},
\end{aligned}
$$

with $t_{s}=t_{0}-\frac{2}{3 H_{0}\left(1+w_{\text {eff, } \infty}\right)}>t_{0}$. This means that the universe has a finite time cosmic singularity in the future, where the total energy density and pressure diverge, i.e., the model has a big rip singularity.

On the other hand, when $\operatorname{Det} A>0$ and $\operatorname{Tr} A>0$, both the eigenvalues are positive, and the origin of coordinates is a repeller, which means that one also obtains a big rip singularity at late times. In order to ensure that the energy densities of the fluids were always positive, one has to impose that origin was not a focus, because if so, at early times the orbits would oscillate around the origin leading to negative energies. To prevent this behavior, one has to impose that the discriminant $\Delta=(\operatorname{Tr} A)^{2}-4 \operatorname{Det} A$ was positive, i.e., the origin was a node. In addition, as we have already explained in Sec. III, for a node, to guarantee the positive values of the energy densities, both the orbits following the respective eigenvectors of the matrix $A$, $\left(X_{+}(N)=e^{\lambda_{+} N} v_{+}\right.$and $X_{-}(N)=e^{\lambda_{-} N} v_{-}$being once again $\lambda_{+}$and $\lambda_{-}$the eigenvalues of the matrix $A$ and, $v_{+}=$ $\left(v_{1,+}, v_{2,+}\right)$ and $v_{-}=\left(v_{1,-}, v_{2,-}\right)$ their corresponding eigenvectors) must belong to the first quadrant, that is, the condition $v_{1, \pm} v_{2, \pm}>0$ must be satisfied. As a consequence, all orbits with an initial condition defined in this region must remain in this region of the first quadrant, which ensures that the energy densities were always positive.

All these conditions lead to the following constraints that the parameters $\mu$ and $\nu$ need to satisfy:

$$
\left\{\begin{array}{l}
2+\mu-\nu+w_{x} \\
(1+\mu)\left(1+w_{x}\right)-\nu>0 \\
\left(w_{x}-\mu-\nu\right)^{2}-4 \mu \nu>0
\end{array}\right.
$$

where the first equation implies that $\operatorname{Tr} A>0$, the second implies $\operatorname{Det} A>0$ and the third ensures $\Delta>0$. Moreover, as we know, the eigenvalues of $A$ are given by $\lambda_{ \pm}=$ $(\operatorname{Tr} A \pm \sqrt{\Delta}) / 2$ and the corresponding eigenvectors can be classified for $\nu \neq 0$ and $\nu=0$ as follows:

(1) For $\nu \neq 0$, the eigenvectors are given by

$$
v_{ \pm}=\left(1,-\frac{\mu+1+\lambda_{ \pm} / 3}{\nu}\right) .
$$

(2) For $\nu=0$, the eigenvalues are $\lambda_{+}=-3\left(1+w_{x}\right)$ and $\lambda_{-}=-3(1+\mu)$, which implies that $w_{x}<-1$ (phantom fluid) and also $\mu<-1$. The eigenvectors are then given by

$$
v_{+}=(0,1), \quad v_{-}=\left(1, \frac{\mu}{w_{x}-\mu}\right) .
$$

Now, the condition $v_{1, \pm} v_{2, \pm}>0$ gives

$$
\left\{\begin{array}{lll}
\frac{\mu+1+\lambda_{ \pm} / 3}{\nu}>0 & \text { for } \quad \nu \neq 0 \\
w_{x} \leq \mu<0 & \text { for } \quad \nu=0 .
\end{array}\right.
$$

Thus, in order that the initial condition was in the region defined by the orbits $X_{+}(N)$ and $X_{-}(N)$, and thus, the energy densities were always positive, we get the following restrictions:

$\min \left(v_{2,+}, v_{2,-}\right) \leq \frac{\rho_{x, 0}}{\rho_{c, 0}} \leq \max \left(v_{2,+}, v_{2,-}\right)$, for $\nu \neq 0$,

and

$$
\frac{\rho_{x, 0}}{\rho_{c, 0}}>\frac{\mu}{w_{x}-\mu}, \quad \text { for } \nu=0
$$

Then, considering the simple case $\nu=0$, and taking into account that $\rho_{x, 0} / \rho_{c, 0}=\Omega_{x, 0} / \Omega_{c, 0}$, and using, once again, the following observational values at present time, namely, $\Omega_{c, 0} \cong 0.262$ and $\Omega_{x, 0} \cong 0.69$, from (47) we deduce that the parameter $\mu$ must satisfy the condition 


$$
0.72 w_{x}<\mu<0
$$

and from (42) we conclude that, to have a future big rip singularity, the value of the parameter $\mu$ has to satisfy

$$
0.72 w_{x}<\mu<-1, \quad \text { with } \quad w_{x}<-1.38 .
$$

With the analysis presented above, it is clear that the linear interaction models may encounter with finite time future singularities (here the big rip singularity) depending on the model parameters, in particular, for some specific regions of the coupling parameter(s) of the interaction function and the dark energy equation of state. Similar to the early time instability problems in the interaction models, as discussed in Sec. III, one can clearly understand that the appearance of finite time future singularities is also related to the choice of interaction function. Since one can construct a number of phenomenological parametrizations of the interaction functions, thus, it is possible to have an interaction model with no finite time singularities in the future. In this connection, we refer to an appealing interacting DE-DM theories where DE acts as a scalar field and the mass of the DM particles has a direct dependence on the scalar field itself [90,91]. In such theories, if the potential and kinetic term of the scalar are well behaved, no future singularity will appear.

\section{CONCLUSIONS}

The theory of nongravitational interaction between DM and DE is one of the fantastic areas of modern cosmology and this is the main theme of this work. Existing articles demand that interacting DE models are one of the promising cosmological models that could explain many theoretical and observational issues related to the evolution of the universe. Being recognized for its ability to soften the cosmological constant problem it came into the limelight for providing with a possible solution to the cosmic coincidence problem. Now, if we concentrate on the recent investigations focused on the tensions in the cosmological parameters arising from local and global measurements, this area has taken a serious role. The readers have already witnessed how the tensions in both $H_{0}$ and $\sigma_{8}$ can be alleviated/solved if an interaction in the dark sector is considered. Therefore, there are enough reasons to select the interacting models as the theme of the present work. In the present article we consider an interacting cosmic scenario between DM and DE driven by a simple interaction rate which is linear in the energy densities of DM and DE. Here, DM has been taken to be pressureless and DE has a constant barotropic equation of state. By performing a systematic dynamical analysis we show explicitly that the inclusion of an interaction in the background may lead to unphysical behaviour in terms of negative energy densities.
To obtain viable cosmic scenarios, one needs to impose additional conditions on the parameter space consisting of the coupling parameters of the interaction rates as well as the DE equation of state. Although the linear interaction models have been investigated widely in the past due to their simplest mathematical structure, but the current article raises some important points that are essential to understand the actual parameter space of the underlying cosmological model.

It is important to mention that the linear interaction models can also lead to finite time future cosmic singularities depending on the model parameters. In particular, we find that for the present interaction model our universe may encounter with a big rip singularity for some specific values of the model parameters. Although the present study focuses on the linear interacting models, but the same can be performed with the nonlinear interaction models. Moreover, we here focus on the simplest case in which DE has a constant barotropic state parameter, however, one could extend the case with dynamical state parameter. In connection with the present interaction model we would like to emphasize on a broad class of interacting DM-DE theories in which DE acts as a scalar field and DM particle has a mass which is directly dependent on the scalar field itself $[90,91]$. This class of interaction theories are very appealing for their far reaching possibilities. In particular, concerning the singularity problem that we faced with the present interaction model can be avoided in the aforementioned theories if the potential and kinetic term of the scalar are well behaved. Thus, it will be interesting to perform a dynamical system analysis of the above interaction models following the similar approach as in the present article, in order to look for viable scenarios both from theoretical and observational grounds. Such investigations will be very enchanting and we believe that, other investigators including us, might be interested to explore the deeper physical insights with such models.

\section{ACKNOWLEDGMENTS}

The authors thank the referees for their useful comments that helped us to improve the quality of discussion of this article. S. P. has been supported by the Mathematical Research Impact-Centric Support Scheme (MATRICS), File No. MTR/2018/000940, given by the Science and Engineering Research Board (SERB), Govt. of India. The investigation of J.dH. has been supported by MINECO (Spain) Grant No. MTM2017-84214-C2-1-P, and in part by the Catalan Government Grant No. 2017-SGR-247. W. Y. was supported by the National Natural Science Foundation of China under Grants No. 11705079 and No. 11647153. The investigation of J. A. has been supported by MINECO (Spain) Grant No. MTM2015-69135-P, and by the Catalan Government Grant No. 2017-SGR-932. 
[1] N. Aghanim et al. (Planck Collaboration), Planck 2018 results. VI. Cosmological parameters, arXiv:1807.06209.

[2] S. Weinberg, The cosmological constant problem, Rev. Mod. Phys. 61, 1 (1989).

[3] C. Wetterich, The cosmon model for an asymptotically vanishing time dependent cosmological 'constant', Astron. Astrophys. 301, 321 (1995).

[4] L. Amendola, Coupled quintessence, Phys. Rev. D 62, 043511 (2000).

[5] L. Amendola and C. Quercellini, Tracking and coupled dark energy as seen by WMAP, Phys. Rev. D 68, 023514 (2003).

[6] R. G. Cai and A. Wang, Cosmology with interaction between phantom dark energy and dark matter and the coincidence problem, J. Cosmol. Astropart. Phys. 03 (2005) 002.

[7] D. Pavón and W. Zimdahl, Holographic dark energy and cosmic coincidence, Phys. Lett. B 628, 206 (2005).

[8] S. del Campo, R. Herrera, and D. Pavón, Toward a solution of the coincidence problem, Phys. Rev. D 78, 021302 (2008).

[9] S. del Campo, R. Herrera, and D. Pavón, Interacting models may be key to solve the cosmic coincidence problem, J. Cosmol. Astropart. Phys. 01 (2009) 020.

[10] A. P. Billyard and A. A. Coley, Interactions in scalar field cosmology, Phys. Rev. D 61, 083503 (2000).

[11] J. D. Barrow and T. Clifton, Cosmologies with energy exchange, Phys. Rev. D 73, 103520 (2006).

[12] L. Amendola, G. Camargo Campos, and R. Rosenfeld, Consequences of dark matter-dark energy interaction on cosmological parameters derived from SNIa data, Phys. Rev. D 75, 083506 (2007).

[13] J. H. He and B. Wang, Effects of the interaction between dark energy and dark matter on cosmological parameters, J. Cosmol. Astropart. Phys. 06 (2008) 010.

[14] J. Väliviita, E. Majerotto, and R. Maartens, Instability in interacting dark energy and dark matter fluids, J. Cosmol. Astropart. Phys. 07 (2008) 020.

[15] M. B. Gavela, D. Hernandez, L. Lopez Honorez, O. Mena, and S. Rigolin, Dark coupling, J. Cosmol. Astropart. Phys. 07 (2009) 034.

[16] E. Majerotto, J. Väliviita, and R. Maartens, Adiabatic initial conditions for perturbations in interacting dark energy models, Mon. Not. R. Astron. Soc. 402, 2344 (2010).

[17] T. Clemson, K. Koyama, G. B. Zhao, R. Maartens, and J. Väliviita, Interacting dark energy-Constraints and degeneracies, Phys. Rev. D 85, 043007 (2012).

[18] S. Pan and S. Chakraborty, Will there be again a transition from acceleration to deceleration in course of the dark energy evolution of the universe?, Eur. Phys. J. C 73, 2575 (2013).

[19] W. Yang and L. Xu, Cosmological constraints on interacting dark energy with redshift-space distortion after Planck data, Phys. Rev. D 89, 083517 (2014).

[20] W. Yang and L. Xu, Testing coupled dark energy with large scale structure observation, J. Cosmol. Astropart. Phys. 08 (2014) 034.

[21] R. C. Nunes and E. M. Barboza, Dark matter-dark energy interaction for a time-dependent EoS parameter, Gen. Relativ. Gravit. 46, 1820 (2014).
[22] V. Salvatelli, N. Said, M. Bruni, A. Melchiorri, and D. Wands, Indications of a Late-Time Interaction in the Dark Sector, Phys. Rev. Lett. 113, 181301 (2014).

[23] S. Pan, S. Bhattacharya, and S. Chakraborty, An analytic model for interacting dark energy and its observational constraints, Mon. Not. R. Astron. Soc. 452, 3038 (2015).

[24] M. Shahalam, S. D. Pathak, M. M. Verma, M. Y. Khlopov, and R. Myrzakulov, Dynamics of interacting quintessence, Eur. Phys. J. C 75, 395 (2015).

[25] N. Tamanini, Phenomenological models of dark energy interacting with dark matter, Phys. Rev. D 92, 043524 (2015).

[26] R. C. Nunes, S. Pan, and E. N. Saridakis, New constraints on interacting dark energy from cosmic chronometers, Phys. Rev. D 94, 023508 (2016).

[27] C. Caprini and N. Tamanini, Constraining early and interacting dark energy with gravitational wave standard sirens: The potential of the eLISA mission, J. Cosmol. Astropart. Phys. 10 (2016) 006.

[28] S. Kumar and R. C. Nunes, Probing the interaction between dark matter and dark energy in the presence of massive neutrinos, Phys. Rev. D 94, 123511 (2016).

[29] W. Yang, H. Li, Y. Wu, and J. Lu, Cosmological constraints on coupled dark energy, J. Cosmol. Astropart. Phys. 10 (2016) 007.

[30] S. Pan and G. S. Sharov, A model with interaction of dark components and recent observational data, Mon. Not. R. Astron. Soc. 472, 4736 (2017).

[31] C. van de Bruck, J. Mifsud, and J. Morrice, Testing coupled dark energy models with their cosmological background evolution, Phys. Rev. D 95, 043513 (2017).

[32] R. Erdem, Is it possible to obtain cosmic accelerated expansion through energy transfer between different energy densities?, Phys. Dark Universe 15, 57 (2017).

[33] G. S. Sharov, S. Bhattacharya, S. Pan, R. C. Nunes, and S. Chakraborty, A new interacting two fluid model and its consequences, Mon. Not. R. Astron. Soc. 466, 3497 (2017).

[34] S. Kumar and R. C. Nunes, Echo of interactions in the dark sector, Phys. Rev. D 96, 103511 (2017).

[35] M. Shahalam, S. D. Pathak, S. Li, R. Myrzakulov, and A. Wang, Dynamics of coupled phantom and tachyon fields, Eur. Phys. J. C 77, 686 (2017).

[36] R. G. Cai, N. Tamanini, and T. Yang, Reconstructing the dark sector interaction with LISA, J. Cosmol. Astropart. Phys. 05 (2017) 031.

[37] E. Di Valentino, A. Melchiorri, and O. Mena, Can interacting dark energy solve the $H_{0}$ tension?, Phys. Rev. D 96, 043503 (2017).

[38] W. Yang, N. Banerjee, and S. Pan, Constraining a dark matter and dark energy interaction scenario with a dynamical equation of state, Phys. Rev. D 95, 123527 (2017).

[39] J. Mifsud and C. Van De Bruck, Probing the imprints of generalized interacting dark energy on the growth of perturbations, J. Cosmol. Astropart. Phys. 11 (2017) 001.

[40] W. Yang, S. Pan, and D. F. Mota, Novel approach toward the large-scale stable interacting dark-energy models and their astronomical bounds, Phys. Rev. D 96, 123508 (2017).

[41] W. Yang, S. Pan, and J. D. Barrow, Large-scale stability and astronomical constraints for coupled dark-energy models, Phys. Rev. D 97, 043529 (2018). 
[42] S. Pan, A. Mukherjee, and N. Banerjee, Astronomical bounds on a cosmological model allowing a general interaction in the dark sector, Mon. Not. R. Astron. Soc. 477, 1189 (2018).

[43] W. Yang, S. Pan, R. Herrera, and S. Chakraborty, Largescale (in) stability analysis of an exactly solved coupled dark-energy model, Phys. Rev. D 98, 043517 (2018).

[44] W. Yang, S. Pan, and A. Paliathanasis, Cosmological constraints on an exponential interaction in the dark sector, Mon. Not. R. Astron. Soc. 482, 1007 (2019).

[45] W. Yang, S. Pan, L. Xu, and D. F. Mota, Effects of anisotropic stress in interacting dark matter C dark energy scenarios, Mon. Not. R. Astron. Soc. 482, 1858 (2019).

[46] R. von Marttens, L. Casarini, D. F. Mota, and W. Zimdahl, Cosmological constraints on parametrized interacting dark energy, Phys. Dark Universe 23, 100248 (2019).

[47] W. Yang, N. Banerjee, A. Paliathanasis, and S. Pan, Reconstructing the dark matter and dark energy interaction scenarios from observations, Phys. Dark Universe 26, 100383 (2019).

[48] A. Paliathanasis, S. Pan, and W. Yang, Dynamics of nonlinear interacting dark energy models, Int. J. Mod. Phys. D 28, 1950161 (2019).

[49] S. Pan, W. Yang, C. Singha, and E. N. Saridakis, Observational constraints on sign-changeable interaction models and alleviation of the $H_{0}$ tension, Phys. Rev. D 100, 083539 (2019).

[50] W. Yang, S. Pan, E. Di Valentino, B. Wang, and A. Wang, Forecasting interacting vacuum-energy models using gravitational waves, arXiv:1904.11980.

[51] W. Yang, S. Vagnozzi, E. Di Valentino, R. C. Nunes, S. Pan, and D.F. Mota, Listening to the sound of dark sector interactions with gravitational wave standard sirens, J. Cosmol. Astropart. Phys. 07 (2019) 037.

[52] W. Yang, O. Mena, S. Pan, and E. Di Valentino, Dark sectors with dynamical coupling, Phys. Rev. D 100, 083509 (2019).

[53] S. Pan, W. Yang, E. Di Valentino, E. N. Saridakis, and S. Chakraborty, Interacting scenarios with dynamical dark energy: Observational constraints and alleviation of the $H_{0}$ tension, Phys. Rev. D 100, 103520 (2019).

[54] J. D. Barrow and G. Kittou, Non-linear interactions in cosmologies with energy exchange, Eur. Phys. J. C 80, 120 (2020).

[55] W. Yang, S. Pan, R. C. Nunes, and D. F. Mota, Dark calling dark: Interaction in the dark sector in presence of neutrino properties after Planck CMB final release, J. Cosmol. Astropart. Phys. 04 (2020) 008.

[56] E. Di Valentino, A. Melchiorri, O. Mena, and S. Vagnozzi, Non-minimal dark sector physics and cosmological tensions, Phys. Rev. D 101, 063502 (2020).

[57] R. von Marttens, L. Lombriser, M. Kunz, V. Marra, L. Casarini, and J. Alcaniz, Dark degeneracy I: Dynamical or interacting dark energy?, Phys. Dark Universe 28, 100490 (2020).

[58] G. Papagiannopoulos, P. Tsiapi, S. Basilakos, and A. Paliathanasis, Dynamics and cosmological evolution in $\Lambda$-varying cosmology, Eur. Phys. J. C 80, 55 (2020).

[59] Y. L. Bolotin, A. Kostenko, O. A. Lemets, and D. A. Yerokhin, Cosmological evolution with interaction between dark energy and dark matter, Int. J. Mod. Phys. D 24, 1530007 (2015).

[60] B. Wang, E. Abdalla, F. Atrio-Barandela, and D. Pavón, Dark matter and dark energy interactions: Theoretical challenges, cosmological implications and observational signatures, Rep. Prog. Phys. 79, 096901 (2016).

[61] B. Wang, Y. G. Gong, and E. Abdalla, Transition of the dark energy equation of state in an interacting holographic dark energy model, Phys. Lett. B 624, 141 (2005).

[62] H. M. Sadjadi and M. Honardoost, Thermodynamics second law and omega $=-1$ crossing(s) in interacting holographic dark energy model, Phys. Lett. B 647, 231 (2007).

[63] S. Pan and S. Chakraborty, A cosmographic analysis of holographic dark energy models, Int. J. Mod. Phys. D 23, 1450092 (2014).

[64] W. Yang, S. Pan, E. Di Valentino, R. C. Nunes, S. Vagnozzi, and D.F. Mota, Tale of stable interacting dark energy, observational signatures, and the $H_{0}$ tension, J. Cosmol. Astropart. Phys. 09 (2018) 019.

[65] W. Yang, A. Mukherjee, E. Di Valentino, and S. Pan, Interacting dark energy with time varying equation of state and the $H_{0}$ tension, Phys. Rev. D 98, 123527 (2018).

[66] S. Kumar, R. C. Nunes, and S. K. Yadav, Dark sector interaction: A remedy of the tensions between $\mathrm{CMB}$ and LSS data, Eur. Phys. J. C 79, 576 (2019).

[67] E. Di Valentino, A. Melchiorri, O. Mena, and S. Vagnozzi, Interacting dark energy after the latest Planck, DES, and $H_{0}$ measurements: An excellent solution to the $H_{0}$ and cosmic shear tensions, arXiv:1908.04281.

[68] C. Van De Bruck and J. Mifsud, Searching for dark matterdark energy interactions: Going beyond the conformal case, Phys. Rev. D 97, 023506 (2018).

[69] A. Pourtsidou and T. Tram, Reconciling CMB and structure growth measurements with dark energy interactions, Phys. Rev. D 94, 043518 (2016).

[70] R. An, C. Feng, and B. Wang, Relieving the tension between weak lensing and cosmic microwave background with interacting dark matter and dark energy models, J. Cosmol. Astropart. Phys. 02 (2018) 038.

[71] C. van de Bruck and J. Morrice, Disformal couplings and the dark sector of the universe, J. Cosmol. Astropart. Phys. 04 (2015) 036.

[72] C. G. Böehmer, N. Tamanini, and M. Wright, Interacting quintessence from a variational approach Part I: Algebraic couplings, Phys. Rev. D 91, 123002 (2015).

[73] C. G. Böehmer, N. Tamanini, and M. Wright, Interacting quintessence from a variational approach Part II: Derivative couplings, Phys. Rev. D 91, 123003 (2015).

[74] J. Gleyzes, D. Langlois, M. Mancarella, and F. Vernizzi, Effective theory of interacting dark energy, J. Cosmol. Astropart. Phys. 08 (2015) 054.

[75] G. D'Amico, T. Hamill, and Nemanja Kaloper, Quantum field theory of interacting dark matter/dark energy: Dark monodromies, Phys. Rev. D 94, 103526 (2016).

[76] S. Pan, G. S. Sharov, and W. Yang, Field theoretic interpretations of interacting dark energy scenarios and recent observations, arXiv:2001.03120.

[77] R. Weitzenböck, Invarianten Theorie (Noordhoff, Groningen, 1923). 
[78] V. C. de Andrade, L. C. T. Guillen, and J. G. Pereira, Teleparallel gravity: An Overview, arXiv:gr-qc/0011087.

[79] B. Li, T. P. Sotiriou, and J.D. Barrow, $f(T)$ Gravity and local Lorentz invariance, Phys. Rev. D 83, 064035 (2011).

[80] A. Unzicker and T. Case, Translation of Einstein's attempt of a unified field theory with teleparallelism, arXiv:physics/ 0503046.

[81] J. de Haro and J. Amorós, Non-Singular Models of Universes in Teleparallel Theories, Phys. Rev. Lett. 110, 071104 (2013).

[82] J. Carminati and R. G. McLenaghan, Algebraic invariants of the Riemann tensor in a four-dimensional Lorentzian space, J. Math. Phys. (N.Y.) 32, 3135 (1991).

[83] A. H. Chamseddine, V. Mukhanov, and A. Vikman, Cosmology with mimetic matter, J. Cosmol. Astropart. Phys. 06 (2014) 017.

[84] A. H. Chamseddine and V. Mukhanov, Mimetic dark matter, J. High Energy Phys. 11 (2013) 135.
[85] P. A. R. Ade et al. (Planck Collaboration), Planck 2015 results. XIII. Cosmological parameters, Astron. Astrophys. 594, A13 (2016).

[86] V. Mukhanov, Physical Foundations of Cosmology (Cambridge University Press, Cambridge, England, 2005).

[87] M. Doran, C. M. Mueller, G. Schaefer, and C. Wetterich, Gauge-invariant initial conditions and early time perturbations in quintessence Universes, Phys. Rev. D 68, 063505 (2003).

[88] Y.-H. Li and X. Zhang, Large-scale stable interacting dark energy model: Cosmological perturbations and observational constraints, Phys. Rev. D 89, 083009 (2014).

[89] G. Olivares, F. Atrio-Barandela, and D. Pavon, Matter density perturbations in interacting quintessence models, Phys. Rev. D 74, 043521 (2006).

[90] C. Wetterich, Cosmologies with variable Newton's Constant', Nucl. Phys. B302, 645 (1988).

[91] A. Pourtsidou, C. Skordis, and E. Copeland, Models of dark matter coupled to dark energy, Phys. Rev. D 88, 083505 (2013). 